\title{
Supplementary Information for: \\ Simulating enhanced methane deliverable capacity of guest responsive pores in intrinsically flexible MOFs
}

\author{
Matthew Witman ${ }^{1}$, Bradley Wright ${ }^{1}$, and Berend Smit ${ }^{1,2 *}$ \\ ${ }^{1}$ Department of Chemical and Biomolecular Engineering, \\ University of California, Berkeley 94720, United States and \\ ${ }^{2}$ Laboratory of Molecular Simulation (LSMO), Institut des Sciences et Ingénierie Chimiques, \\ Valais, Ecole Polytechnique Fédérale de Lausanne (EPFL), \\ Rue de l'Industrie 17, CH-1951 Sion, Switzerland
}

\section{S1. FORCE FIELD DETAILS}

The Universal Force Field (UFF) was used to describe all framework bonded and non-bonded interactions [1], while the united atom TraPPE model was used for the methane interactions. MOF/methane interaction parameters were determined by Lorentz-Berthelot combining rules. The automated UFF force field assignment was performed using the LammpsInterface package [2]. For convenience the "fix-metal" approximation was used, which fixes the equilibrium distance of all metal-oxygen bonds to the distance detected in experimental framework and then assigns the harmonic spring constant value according to UFF prescribed rules. More details on this procedure can be found elsewhere $[2,3]$. The RASPA and Lammps input files specifying the force field parameters are given in the supporting simulation files.

\section{S2. SIMULATION DETAILS}

The Lammps package was used to run MD simulations [4]. For each loading state of $0 \leq N<N_{\max }$, the MOF structure was loaded with the specified number of adsorbates and a brief MC simulation using RASPA was performed in the NVT ensemble to resolve overlaps [5]. An MD simulation was then run, using the adsorbate loaded MOF as the starting configuration, at $300 \mathrm{~K}$ with $50 \mathrm{ps}$ of equilibration followed by $100 \mathrm{ps}$ of production with integration time steps of $1 \mathrm{fs}$ using the Nose-Hoover thermostat. A simulation snapshot was saved every $50 \mathrm{fs}$. Again using the RASPA package, 20 Widom moves were performed on each snapshot to accumulate the unbiased adsorbate insertion/deletion acceptance probabilities from which the C-matrix, transition probabilities, and macrostate probabilities can be computed. The sampling procedure in terms of the quantities referenced in Algorithm 1 are shown in Table I.

\begin{tabular}{c|c} 
parameter & value \\
\hline$N_{\max }$ & 80 \\
$L$ & $100 \mathrm{ps}$ \\
$s$ & $50 \mathrm{fs}$ \\
$w$ & 20
\end{tabular}

TABLE I. NVT(MD)+W sampling parameters for methane in teMOF-5.

\section{S3. DIFFUSION COEFFICIENT CALCULATIONS}

Diffusion coefficients of methane in teMOF-5 can be computed from the same MD simulations that were used to generate the full isotherm from the NVT(MD)+W sampling procedure. The convergence of the MSD from these trajectories is shown in Figure 1. While the NVT(MD)+W sampling procedure generates data from which both the fully flexible isotherm and the loading dependent diffusion coefficients can be calculated, significantly more sampling is required to compute diffusion coefficients. The entire fully flexible isotherm can be calculated with an MD trajectory of $L \sim 100$ ps at each $N$ state, whereas $L \sim 2$ ns is required to compute the diffusion coefficient at each $\mathrm{N}$ state, as can be seen from Figure 1.

\footnotetext{
*berend.smit@epfl.ch
} 


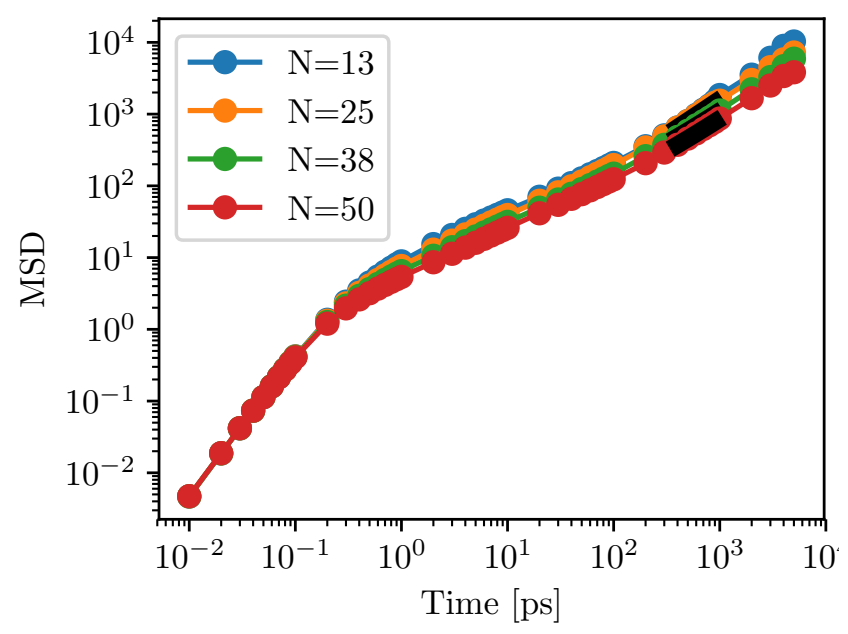

FIG. 1. Convergence of the MSD for MD simulations at different $N$ values. Bold black lines show the regime of the MSD vs time in which the slope is unity on a $\log -\log$ scale and from which the diffusion coefficient can be calculated.

\section{S4. SUPPORTING SIMULATION FILES}

The supporting Lammps and RASPA simulation files are provided in supporting_files.zip. The RASPA input file simulation.input contains an MC move not provided in the default installation, listed in line 23 as SwapGhostProbability. To implement this move, one simply can copy the SwapProbability routine and modify it to write $\operatorname{acc}\left(\Gamma_{N} \rightarrow \Gamma_{N+1}\right)$ and $\operatorname{acc}\left(\Gamma_{N} \rightarrow \Gamma_{N-1}\right)$ rather than actually accepting the particle insertion/deletion, i.e. Swap GhostProbability simply writes the results of a Widom insertion and deletion move.

[1] A. K. Rappe, C. J. Casewit, K. S. Colwell, W. A. Goddard, and W. M. Skiff, UFF, a full periodic table force field for molecular mechanics and molecular dynamics simulations, J. Am. Chem. Soc. 114, 10024 (1992).

[2] P. G. Boyd, S. M. Moosavi, M. Witman, and B. Smit, Force-Field Prediction of Materials Properties in Metal-Organic Frameworks, J. Phys. Chem. Lett. 8, 357 (2017).

[3] M. Witman, S. Ling, S. Jawahery, P. G. Boyd, M. Haranczyk, B. Slater, and B. Smit, The Influence of Intrinsic Framework Flexibility on Adsorption in Nanoporous Materials, J. Am. Chem. Soc. 139, 5547 (2017).

[4] S. Plimpton, Fast Parallel Algorithms for Short-Range Molecular Dynamics, J. Comput. Phys. 117, 1 (1995).

[5] D. Dubbeldam, S. Calero, D. E. Ellis, and R. Q. Snurr, RASPA: molecular simulation software for adsorption and diffusion in flexible nanoporous materials, Mol. Simul. 42, 81 (2016). 